\title{
C-terminal domain of nonhistone protein HMGB1 as a modulator of HMGB1-DNA structural interactions
}

\author{
E. Chikhirzhina ${ }^{\mathrm{a}, *, * *}$, A. Polyanichko ${ }^{\mathrm{a}, \mathrm{b}, *}$, Z. Leonenko ${ }^{\mathrm{c}, * * *}, \mathrm{H}$. Wieser $^{\mathrm{c}}$ and V. Vorob'ev $^{\mathrm{a}}$ \\ ${ }^{a}$ Institute of Cytology, Russian Academy of Sciences, St. Petersburg, Russia \\ ${ }^{\mathrm{b}}$ Department of Molecular Biophysics, Faculty of Physics of St. Petersburg State University and \\ Institute of Cytology RAS, St. Petersburg, Russia \\ ${ }^{\mathrm{c}}$ Department of Chemistry, University of Calgary, Calgary, Alberta, Canada
}

\begin{abstract}
The HMGB1 protein (High Mobility Group protein 1) participates in the formation of functionally significant DNAprotein complexes. HMGB1 protein contains two DNA-binding domains and negatively charged C-terminal region. The latter consists of continuous sequence of dicarboxylic amino acids residues. Structural changes in DNA-protein complexes were studied by circular dichroism spectroscopy (CD) and atomic force microscopy (AFM). Natural HMGB1 and recombinant protein HMGB1 $(\mathrm{A}+\mathrm{B})$ lacked negatively charged C-terminal region were used. The DNA-HMGB1 $(\mathrm{A}+\mathrm{B})$ complexes demonstrate an unusually high optical activity in $150 \mathrm{mM} \mathrm{NaCl}$ solutions. AFM of the latter complexes shows, that at the low concentration of HMGB1 in the complex the protein is distributed along DNA in a random way. Increase of HMGB1 content leads to cooperative interaction and a redistribution of the bound protein molecules on DNA is observed. Based on the data obtained we conclude that protein-protein interactions play a key role in the formation of highly ordered DNA-HMGB1 complexes. It was shown that C-terminal domain modulate the interactions of DNA with HMGB1 protein. We suggest that the C-terminal domain of HMGB1 also modulates the "packing" of HMGB1 molecules on the DNA.
\end{abstract}

Keywords: DNA, chromatin, HMGB1, circular dichroism, VCD

\section{Introduction}

Structural-functional relationships in chromatin have been studied for many years. Among numerous DNA-protein complexes determining chromatin functioning [4] the most intriguing ones in many respects are those between DNA and HMGB-domain proteins. HMGB-proteins are the members of a large family of HMG (High Mobility Group) non-histone chromatin proteins [1,2,11]. Characteristic feature of the proteins within the HMGB subfamily is the presence of structural-functional domain, with very unusual DNA-binding properties, called HMGB-domain (HMG-Box) [2]. HMGB-proteins are the most abundant non-histone proteins found in all studied eukaryotes. The members of the family were

\footnotetext{
*The authors are contributed equally to the preparation of the manuscript.

** Corresponding author: Dr. Elena Chikhirzhina, Senior Research Scientist at the Laboratory of Cell Biochemistry, Institute of Cytology of the Russian Academy of Sciences, 4 Tikhoretsky Ave., St. Petersburg, 194064, Russia. Tel.: +7 812297 3740; Fax.: +7 812297 0341; E-mail: chikhir@gmail.com.

*** Current address: Department of Physics \& Astronomy, University of Waterloo, 200 University Avenue West, Waterloo, Ontario, Canada N2L 3G1.
} 
identified among transcriptional and regulatory factors of chromatin $[1,2,11]$. The typical representative of the HMGB-proteins is HMGB1. This protein contains two almost identical HMGB-domains and C-terminal sequence of 30 dicarboxylic amino acid residues [2]. We expect that the latter negatively charged sequence affects the manner of protein-DNA interaction. In this study we compared the interaction of two proteins HMGB1 itself and a recombinant protein HMGB1 $(\mathrm{A}+\mathrm{B})$, containing both DNA-binding HMGB-domains, but lacking the C-terminal charged sequence.

\section{Materials and methods}

HMGB1 (MW 26500) isolated from calf thymus as described earlier [3] and recombinant protein HMGB1(A + B) (MW 19152) obtained in E. coli [8] were used. Calf thymus DNA (Sigma) was characterized as describes earlier [10] and used without further purification. All aqueous solutions were prepared using double-distilled water. The protein content in the system was described in terms of the protein to DNA weight to weight ratio $(r, \mathrm{w} / \mathrm{w})$, which varied from 0 to 2 . In terms of molar ratio of protein to DNA base pairs $R$, the w/w ratio $r=1$ corresponds to $R=1: 40$ for HMGB1 and $R=1: 29$ for HMGB1 $(\mathrm{A}+\mathrm{B})$. All the complexes were studied in low ionic strength $(15 \mathrm{mM} \mathrm{NaCl})$ and high ionic strength $(150 \mathrm{mM} \mathrm{NaCl})$ conditions.

UV absorbance and ECD spectra of the complexes were recorded with a Jasco-715 spectropolarimeter (Jasco Corp., Japan) in $1 \mathrm{~mm}$ cylindrical quartz cells; concentration of DNA in the samples was $0.3 \mathrm{mg} / \mathrm{ml}$. The detailed description of the spectroscopic approach is given elsewhere [7]. The AFM images were obtained on mica in a tapping mode with PicoSPM (Molecular Imaging, MO, USA) instrument.

\section{Results and discussion}

In our previous studies [3] we have shown that depending on HMGB1 to DNA ratio $r$ one can identify several stages of interaction between HMGB1 and DNA characterized by unique structural reorganization of the complexes. The most relevant for spectroscopic studies are the complexes with relatively low protein to DNA ratios, where no aggregation events occur in the solutions. Based on the previous results, we expect that the mode of interaction between HMGB-domains and DNA is affected by the negatively charged C-terminal region of HMGB1. If this is true, than the HMGB1-DNA interaction should also depend on the concentration of the counter-ions in the solution. To check this assumption we prepared complexes of DNA with intact HMGB1 and with the recombinant protein HMGB1 $(\mathrm{A}+\mathrm{B})$ lacking the charged $\mathrm{C}$-terminal sequence. Figures 1-3 show the CD spectra of DNA-HMGB1 and DNAHMGB1(A + B) at different protein to DNA ratios $r$ in solutions of low (Fig. 1) and high (Figs 2 and 3) ionic strength. The CD spectra of the DNA-protein complexes contain two spectral regions divided by the wavelength $\lambda \approx 250 \mathrm{~nm}$. One of them is located in the region 200-250 nm and represents spectral features attributed to optical activity of both DNA and the proteins, while another one, between 250 and $320 \mathrm{~nm}$, corresponds to the optical activity of DNA only.

According to the CD spectra (Fig. 1) the manner of interaction of these two proteins with DNA differs in the solutions of lower ionic strength. It can be clearly seen (Fig. 1A) that no significant changes in DNA structure can be observed in the HMGB1-DNA complexes up to $r=0.5(R=1: 80)$. In fact, the CD spectra of the HMGB1-DNA complexes at $r<0.5$ demonstrate no significant changes in the secondary structure of either protein or DNA and can be represented by a simple sum of the CD 

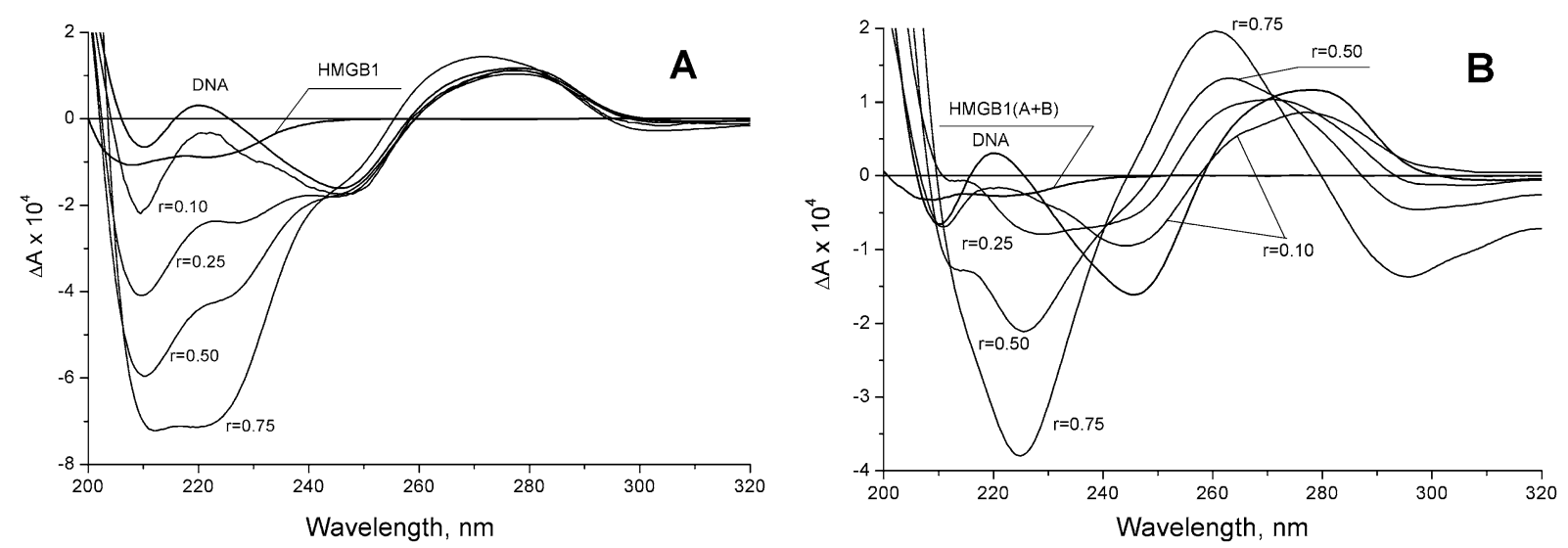

Fig. 1. CD spectra of DNA complexes with non-histone chromosomal protein HMGB1 (A) and recombinant protein HMGB1 $(\mathrm{A}+\mathrm{B})(\mathrm{B})$ in $15 \mathrm{mM} \mathrm{NaCl}$ at different protein to DNA ratios $(r, \mathrm{w} / \mathrm{w}): 0.1 ; 0.25 ; 0.5$ and 0.75 .
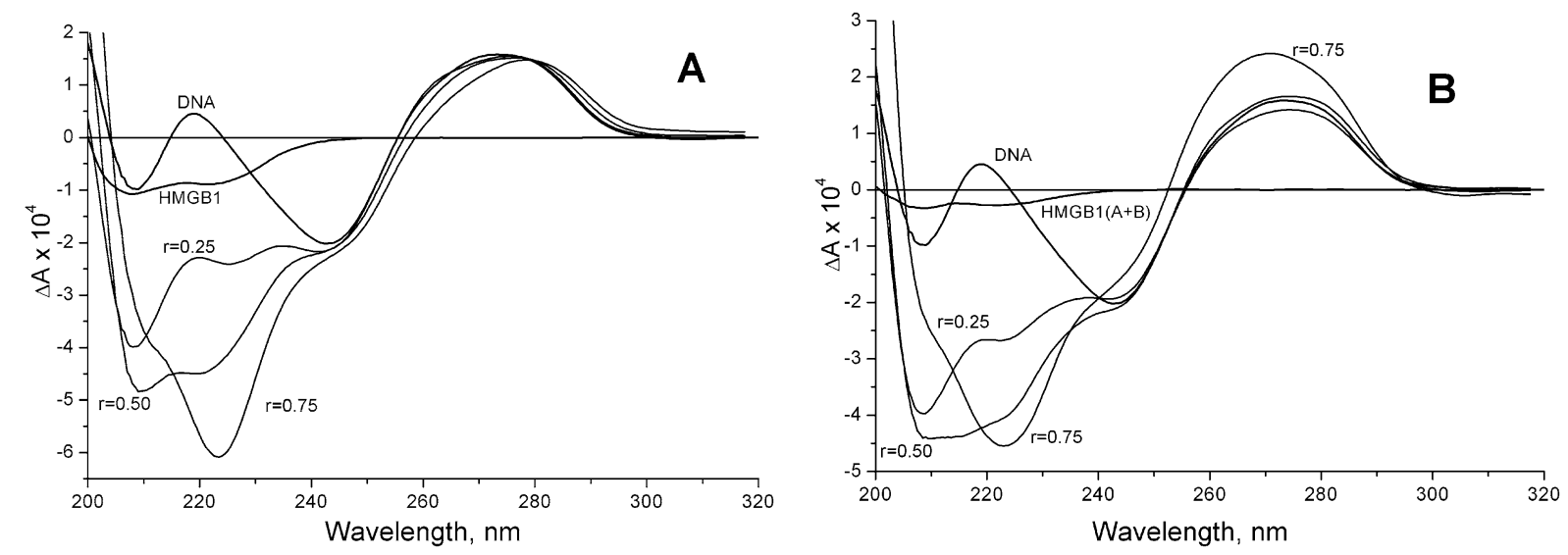

Fig. 2. CD spectra of DNA complexes with non-histone chromosomal protein HMGB1 (A) and recombinant protein HMGB1 $(\mathrm{A}+\mathrm{B})(\mathrm{B})$ in $150 \mathrm{mM} \mathrm{NaCl}$ at different protein to DNA ratios $(r, \mathrm{w} / \mathrm{w}): 0.25 ; 0.5$ and 0.75 .
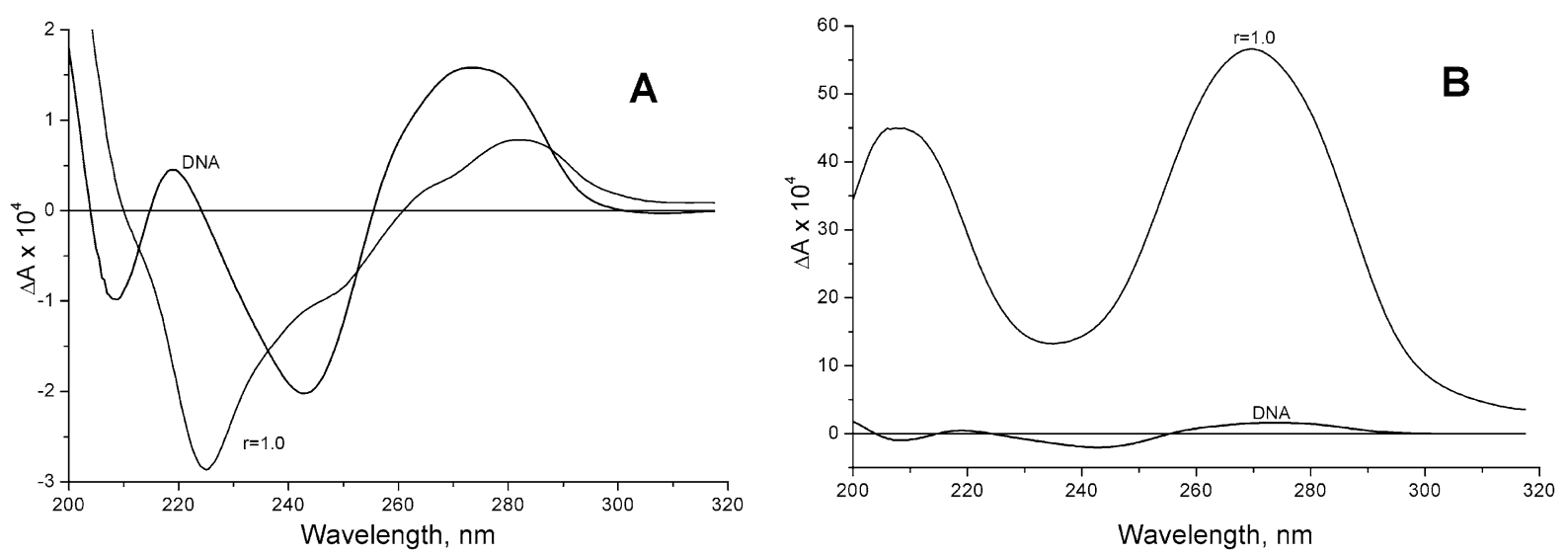

Fig. 3. CD spectra of DNA complexes with non-histone chromosomal protein HMGB1 (A) and recombinant protein $\mathrm{HMGB} 1(\mathrm{~A}+\mathrm{B})(\mathrm{B})$ in $150 \mathrm{mM} \mathrm{NaCl}$ at protein to DNA ratio $(r, \mathrm{w} / \mathrm{w}) r=1.0$. 

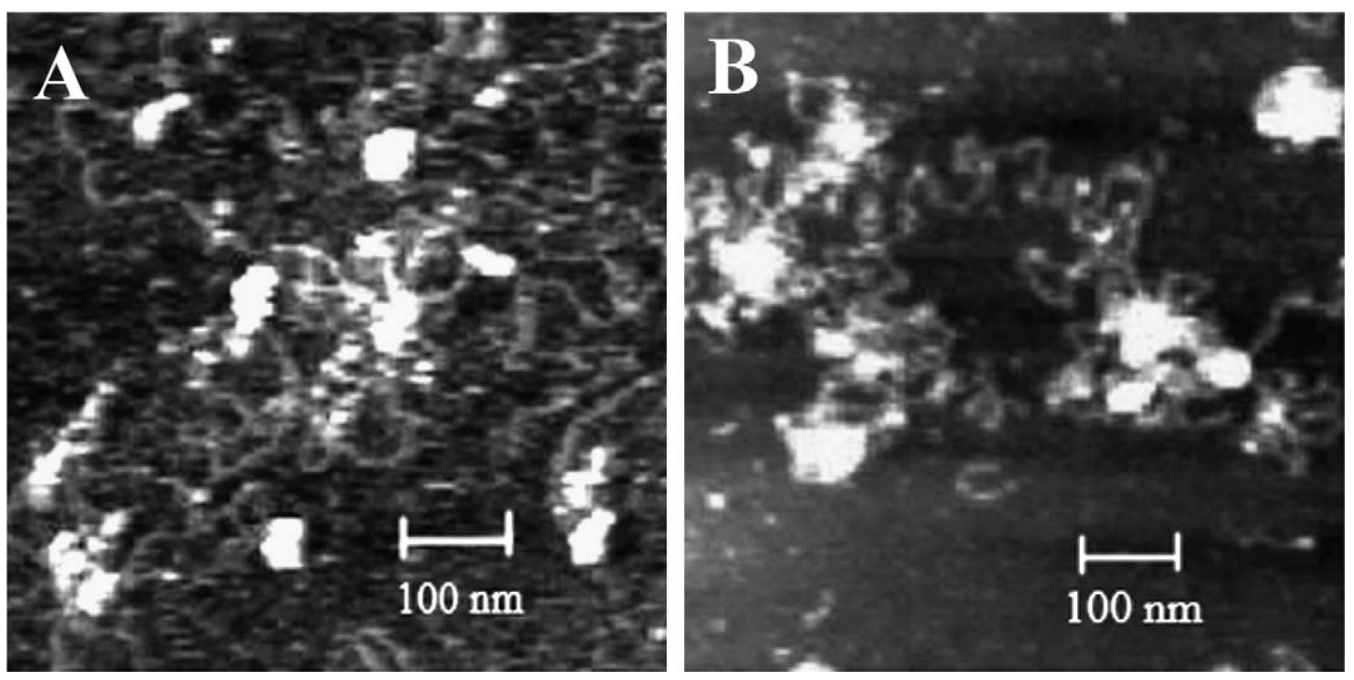

Fig. 4. Atomic Force Microscopy images of the complexes between DNA and chromosomal protein HMGB1 (A) and recombinant protein HMGB1(A + B) (B) prepared in solutions of $15 \mathrm{mM} \mathrm{NaCl}$ at protein to DNA ratio $(\mathrm{w} / \mathrm{w}) r=0.5$. The images are obtained in a tapping mode on mica.

spectra of the individual components. The higher HMGB1 concentrations induce light scattering in the solutions indicating the beginning of co-operative stage of interaction [3] resulting in aggregation, which can be noticed in the spectra corresponding to $r=0.75(R=1: 60)$. The situation is different for the HMGB1(A + B)-DNA complexes (Fig. 1B). Even at $r=0.1$, i.e. one protein molecule to $\sim 290$ b.p. ( $R=1: 290)$, the spectra demonstrate strong changes in CD of DNA, indicating that the recombinant HMGB1 $(A+B)$ interacts with DNA easier than its natural analog HMGB1. The first indications of the formation of large light scattering complexes can be seen at $r=0.25(R \sim 1: 115)$ in this case, which is twice smaller compared to the full-sized HMGB1 (Fig. 1A). This can be explained if one assume that in the HMGB1(A + B)-DNA complexes both HMGB-domains are able to bind DNA. With some probability they could bind distant regions of the same DNA molecule or even different molecules of DNA forming intramolecular clips or intermolecular linkages, which could explain the appearance of light scattering in the solutions at lower protein concentrations. This idea is illustrated by AFM images, obtained for the complexes with $r=0.5$ (Fig. 4). The HMGB1-DNA complexes (Fig. 4A) have a condensed central part with rather extent fragments of DNA coming out of it in different directions. This picture is typical for DNA condensation events and represents the first stage of the formation of larger DNA-protein complexes. For the HMGB1(A + B)-DNA complexes (Fig. 4B) the picture is different. From the one hand, there are similar condensed regions of DNA in the complexes, but from the other hand they are not the central parts of the complexes any more. They are randomly distributed between highly looped DNA regions. To our opinion, the latter resemble most of all the protein clips on a long DNA molecule.

If the C-terminal tail of the HMGB1 protein is just a steric hindrance, then increasing ionic strength of the solvent should not noticeably affect the interaction, and HMGB1 $(\mathrm{A}+\mathrm{B})$ will bind DNA much easier than the HMGB1. On the contrary, if the difference is conditioned by the negative charge of the protein tail, then an increase in the concentration of the counter-ions by an order of magnitude will result in screening the negative charges of both phosphate groups of DNA and carboxylic groups of the protein. In such a case the manner of the interaction of HMGB1 and HMGB1(A + B) with DNA should be 
similar. Figure 2 shows the CD spectra of the HMGB1-DNA (Fig. 2A) HMGB1(A + B)-DNA (Fig. 2B) complexes at moderate protein to DNA ratios $(r<0.75)$ in presence of $150 \mathrm{mM} \mathrm{NaCl}$. In contrast to the spectra of the solutions of lower ionic strength (Fig. 1) the CD spectra of the complexes of DNA with the both proteins give qualitatively similar picture in $150 \mathrm{mM} \mathrm{NaCl}$ (Fig. 2). According to the $\mathrm{CD}$ spectra, neither HMGB1, nor HMGB1 $(\mathrm{A}+\mathrm{B})$ induces serious changes in DNA structure, although they demonstrate some spectral variations in protein optical activity. The first sign of light scattering in the solutions in this case can be observed at $r=0.75$, i.e. $R=1: 60$ for HMGB1 and a little earlier than at $R=1: 44$ for HMGB1 $(\mathrm{A}+\mathrm{B})$ complexes. Thus we may conclude that the increasing ionic strength weakened the HMGB1 $(\mathrm{A}+\mathrm{B})$ interactions with DNA, and seems to have no effect on the HMGB1-DNA interaction. The former is quite expected due to the electrostatic nature of the DNA-protein interactions in general, while the latter is not so clear for the same reason. Most likely the behavior of HMGB1DNA systems is more complex than a simple electrostatic effect. It is possible, that the charged tail of the protein prevented one of the HMGB-domains from the interaction with DNA in solutions with lower ionic strength. Screening of the tail at higher ionic strength could allow both domains of the protein to interact with DNA that countervailed the weakening of the interaction of the individual HMGBdomains. Increasing protein to DNA ratio in the complex induces co-operative binding of the proteins, which results in an aggregation of HMGB1-DNA complexes, strong light scattering and $\psi$-type of CD spectra (Fig. 3A) [5,6]. However, complexes of DNA with HMGB1 $(A+B)$ give a very strong CD signal (Fig. 3B), similar to that of highly ordered DNA structures [9]. The corresponding AFM image is given in Figure 5. The exact structure of the complex is not very clear from the image, however one can see that the core part of it is represented by a rod-like complexes approximately $300 \times 50 \mathrm{~nm}$.

We do not have enough information to discuss the detailed structural organization of such complexes, which require additional structural experiments. Nevertheless, we can assume that the formation of these rod-shaped structures requires higher level of structural order of DNA and protein molecules in the complex. And this level of structural order was achieved in complexes of DNA with HMGB1(A + B), but not with HMGB1 protein. Perhaps, the reason for that is the steric hindrance of the C-terminal tail of HMGB1, mentioned above. It is also possible, that the structural order in the complex also requires a
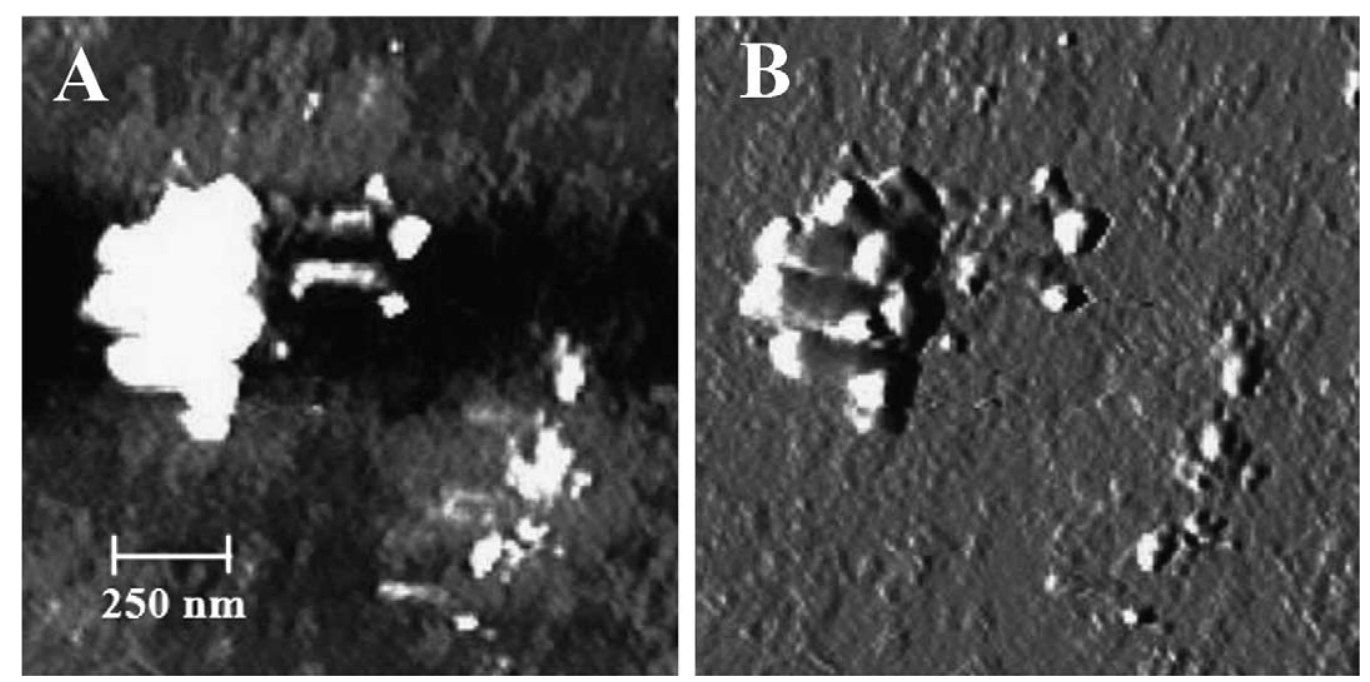

Fig. 5. AFM height trace (A) and phase trace (B) images of DNA-HMGB1 $(A+B)$ complexes taken in a tapping mode on mica. The complexes were prepared in solutions of $150 \mathrm{mM} \mathrm{NaCl}$. Protein to DNA ratio $(r, \mathrm{w} / \mathrm{w}) r=1$. 
higher density of the spatial packaging of the molecules, which is more difficult for the polymer chains with high density of the charge, even at higher ionic strength. In any case, the presence of highly negatively charged C-terminal sequence in HMGB1 strongly affects its interaction with DNA. This effect depends on the ionic strength of the solution and has a different influence on the different HMGB-domains of the protein. Thus, we believe that the C-terminal domain of HMGB1 protein might act as a negative regulator of DNA-protein interaction.

\section{Acknowledgements}

The work was supported by the Russian Foundation for Basic Research (Grant 09-08-01119), Government of St. Petersburg (A. Polyanichko, E. Chikhirzhina) and NSERC (H. Wieser). The authors express their special gratitude to the Department of Chemistry at the University of Calgary for providing the instrumentation for UV and ECD spectroscopy; personally to Dr. David Cramb, for providing instrumentation for AFM imaging. We are also grateful to Elena Kostyleva (Institute of Cytology, RAS) for her help in protein purification. The authors are very grateful to Prof. Colyn Crane-Robinson (Portsmouth, UK) for plasmid phsHMG1/7-170/4.

\section{References}

[1] M. Bustin, Regulation of DNA-dependent activities by the functional motifs of the High-Mobility-Group chromosomal proteins, Mol. Cell Biol. 19 (1999), 5237-5246.

[2] M. Bustin and R. Reeves, High-mobility-group chromosomal proteins: architectural components that facilitate chromatin function, Proc. Natl. Acad. Sci. USA 54 (1996), 35-100.

[3] E.V. Chikhirzhina, A.M. Polyanichko, A.N. Skvortsov, E.V. Kostyleva, C. Houssier and V.I. Vorob'ev, HMG1 domains: The victims of circumstances, Mol. Biol. 36 (2002), 525-531.

[4] E.V. Chikhirzhina and V.I. Vorob'ev, Linker histones: conformational changes and the role in the structural organization of chromatin, Tsitologiia 44 (2002), 721-736.

[5] C.F. Jordan, L.S. Lerman and J.H. Venable, Structure and circular dichroism of DNA in concentrated polymer solutions, Nat. New Biol. 236 (1972), 67-70.

[6] L.S. Lerman, A transition to a compact form of DNA in polymer solutions, Proc. Natl. Acad. Sci. USA 68 (1971), 18861890.

[7] A.M. Polyanichko, V.V. Andrushchenko, E.V. Chikhirzhina, V.I. Vorob'ev and H. Wieser, The effect of manganese(II) on DNA structure: electronic and vibrational circular dichroism studies, Nucl. Acids Res. 32 (2004), 989-996.

[8] C. Read, P. Cary, C. Crane-Robinson, P. Driscoll and D. Norman, Solution structure of a DNA-binding domain from HMG1, Nucl. Acids Res. 21 (1993), 3427-3436.

[9] V.I. Salianov, V.S. Prasolov, M. Palumbo and I.M. Evdokimov, Anomalous optical activity induced during condensation of various spatial forms of double-stranded circular DNA, Biofizika 34 (1989), 20-27.

[10] A.S. Spirin, Spectrophotometric determination of total nucleic acids, Biokhimiia 23 (1958), 656-662.

[11] A. Travers and J.O. Thomas, Chromosomal HMG-box proteins, in: Chromatin Structure and Dynamics: State-of-the-Art, J. Zlatanova and S.H. Leuba, eds, Elsevier, New York, 2004, pp. 103-126. 


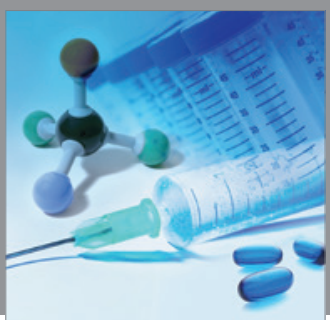

International Journal of

Medicinal Chemistry

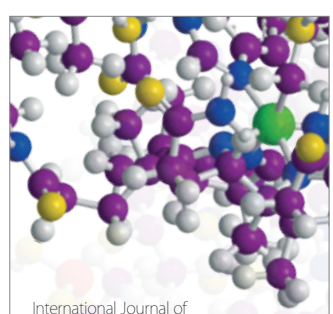

Carbohydrate Chemistry

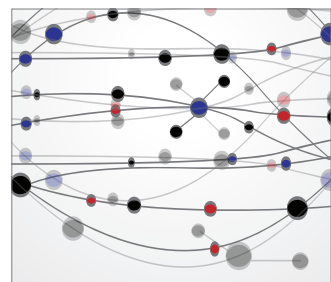

The Scientific World Journal
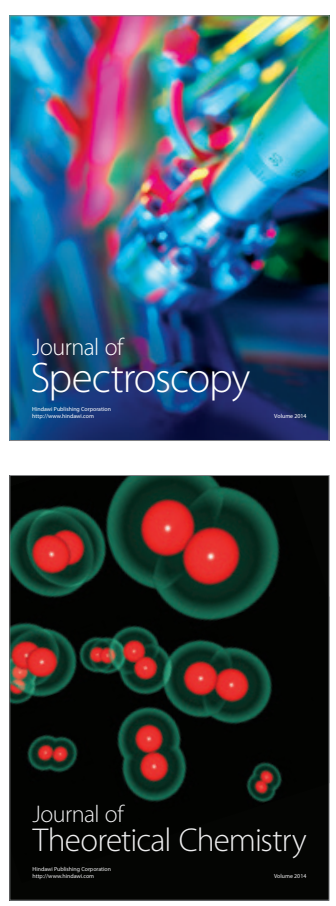
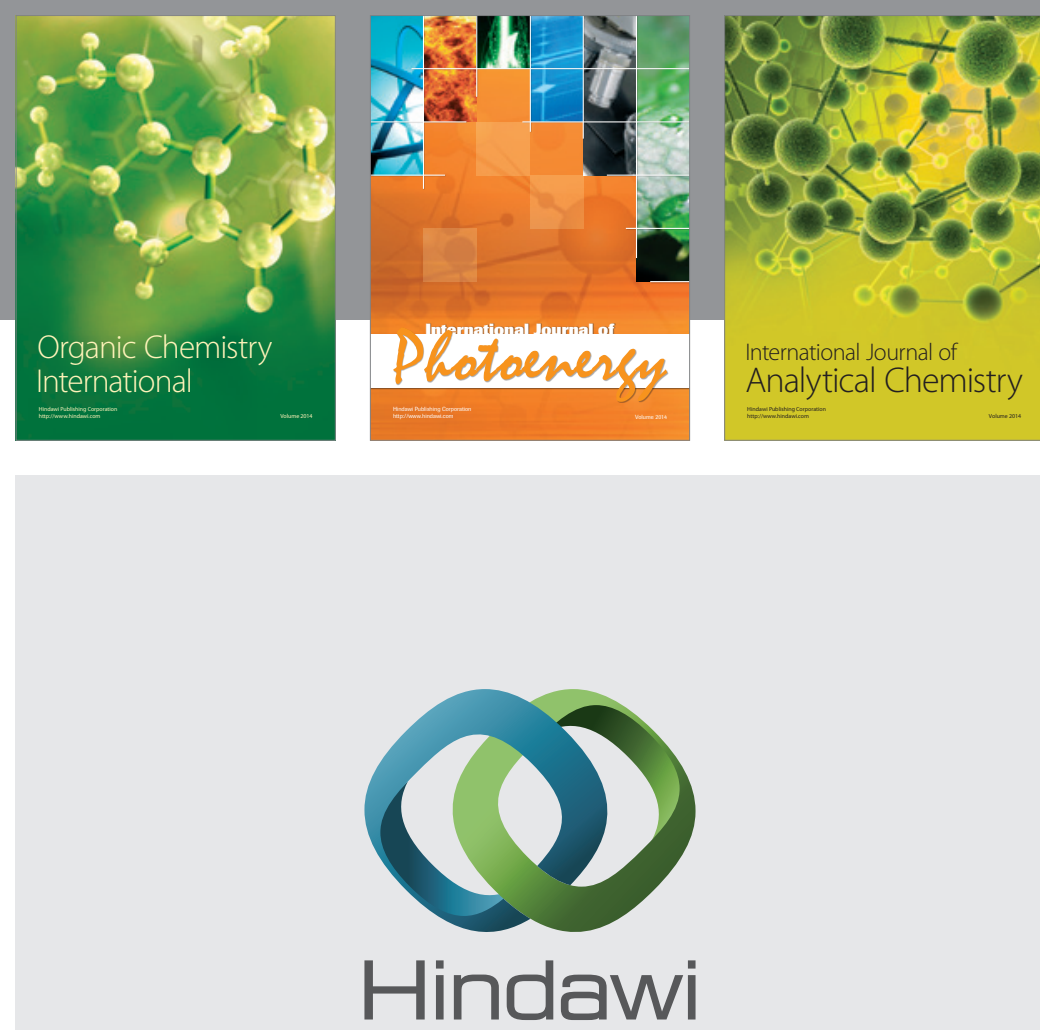

Submit your manuscripts at

http://www.hindawi.com
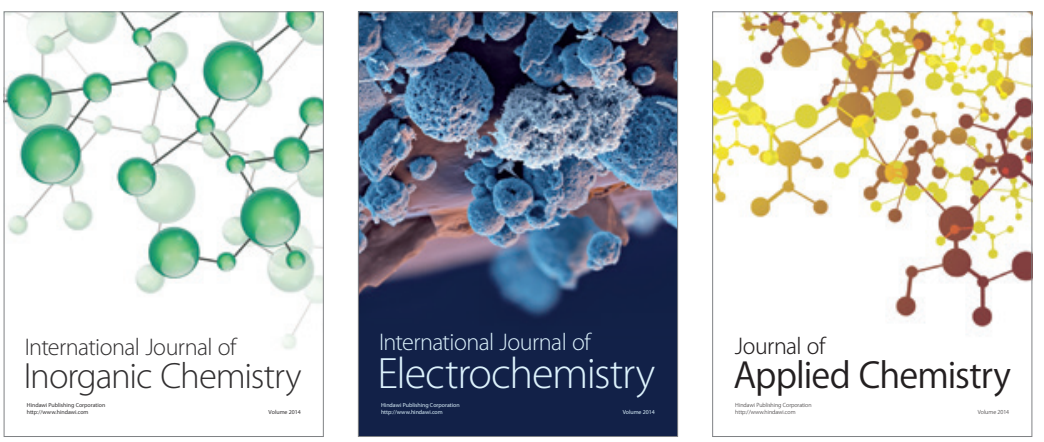

Journal of

Applied Chemistry
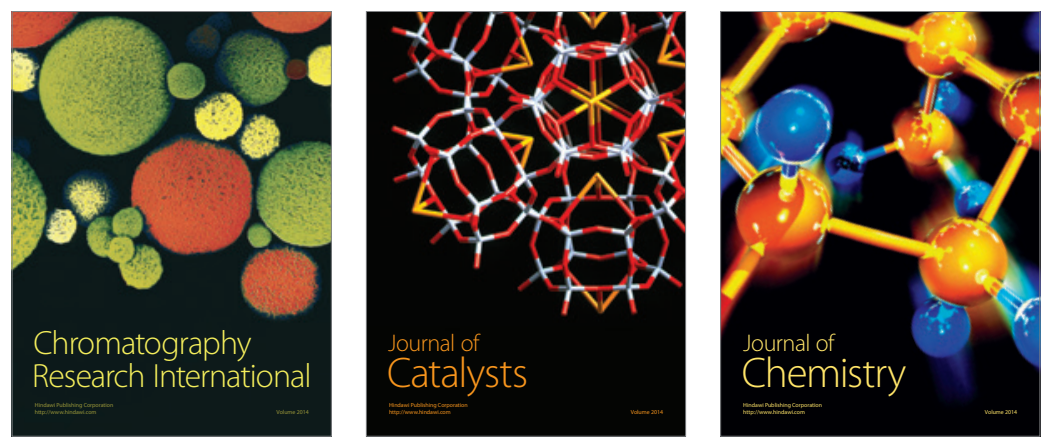
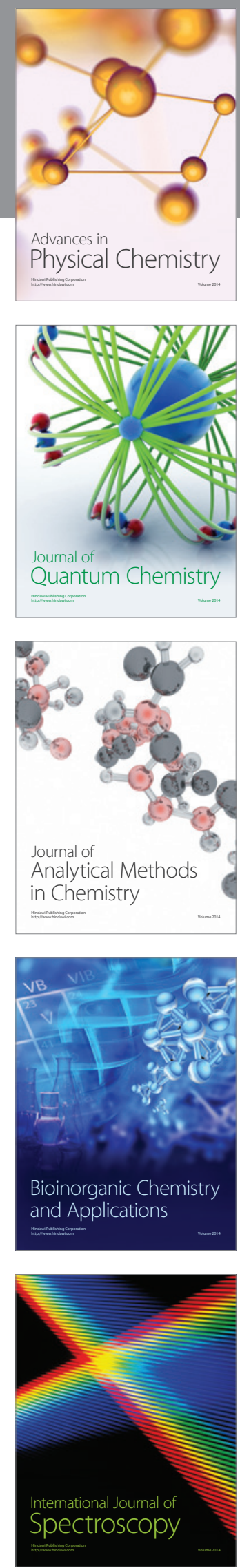